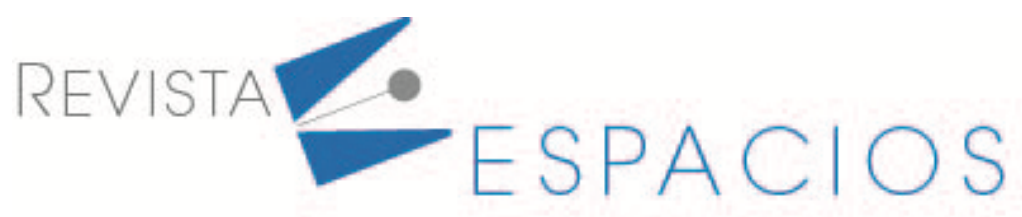

\title{
Accesibilidad geográfica y construcción de paz: un acercamiento desde el desarrollo de la red de infraestructura vial
}

\section{Geographical accessibility and peace construction: an approach from the development of road infrastructure network}

\author{
CARDONA, Santiago ${ }^{1}$ \\ ESCOBAR, Diego A. ${ }^{2}$ \\ HENAO, Edison ${ }^{3}$
}

\begin{abstract}
Resumen
Este articulo de investigación tiene como objetivo determinar la relación entre la accesibilidad geográfica y el conflicto armado en el departamento de Caldas. Se usaron capas y bases de datos abiertas por entidades estatales colombianas y la DistanceMatrixApi de Google para la validación y calibración de la red de infraestructura vial. Se encontró que las zonas rurales, históricamente afectadas por el conflicto, poseen una accesibilidad más baja, es decir, peores conexiones a través de la red vial en el departamento.

Palabras clave: accesibilidad geográfica, conflicto armado, distancematrix api, paz.

Abstract

This research article seeks to determinate the relationship between geographical accessibility and armed conflict in Caldas's department. Using open geographical shapes and databases from Colombian National entities and the DistanceMatrixApi of Google for calibration and validation of the road infrastructure network. Research found that rural zones, historically affected by armed conflict, has low accessibility indicators consequently worse connexions through road network in the department.

key words: geographical accessibility, armed conflict, distancematrix api, peace.
\end{abstract}

\section{Introducción}

El conflicto armado en Colombia ha generado enormes afectaciones a la población y al desarrollo del país, especialmente en las zonas rurales remotas, dónde el abandono estatal generó que las guerrillas se asentaran en más de seis décadas que ha perdurado el conflicto armado más largo de la historia moderna en el mundo (Grupo de Memoria Histórica, 2013). Con la firma del tratado de paz entre las FARC-EP, la guerrilla más grande y poderosa y el gobierno nacional en 2016 se espera que se inicie una transición hacia el postconflicto y

\footnotetext{
${ }^{1}$ Jóven Investigador. MSC en Ingeniería - Infraestructura y Sistemas de Transportes. Departamento de Ingeniería y Arquitectura. Universidad Nacional de Colombia sede Manizales. Email: scardonau@unal.edu.co

2 PhD en Gestión del Territorio e Infraestructuras del Transporte. Universidad Nacional de Colombia, Sede Manizales. Facultad de Ingeniería y Arquitectura, Departamento de Ingeniería Civil, Grupo de investigación en Movilidad Sostenible. Campus La Nubia, Bloque S2-208, Carrera 37 con Calle 94, Manizales, 170003, Colombia. (email: daescobarga@unal.edu.co)

${ }^{3}$ Phd, Universidad Nacional de Colombia, Sede Manizales. Facultad de Ingeniería y Arquitectura, Escuela de Arquitectura y Urbanismo. Carrera 23 \# 65, Manizales, 170004, Manizales, Colombia. (email: ehenaoc@unal.edu.co).
} 
construcción de paz, generándose inversión y mayor atención a las zonas rurales del país con el objetivo de cerrar la brecha de desarrollo potenciada por la presencia del conflicto armado en sus territorios (Calderón Rojas, 2016; Presidencia de la República \& FARC-EP, 2016; Sandino, Simanca, Páez, Cortéz-Méndez, Palacios-Rozo, \& Alfaro, 2019).

\subsection{Breve historia del conflicto armado y su incidencia en el territorio}

La inciden del conflicto armado en los territorios variaron en intensidad a lo largo de los años, impulsadas por diferentes motivaciones políticas y económicas. La fundación de las guerrillas (FARC, ELN, EPL) se da en la década de los sesenta, luego de un periodo conocido como "La Violencia" dónde los dos partidos políticos tradicionales (Liberales y Conservadores) pelearon en una "guerra civil" que dejó numerosas víctimas, principalmente en la zona andina y terminó con la conformación del Frente Nacional (1958-1974), un periodo de 16 años donde ambos partidos se turnarían el poder cada 4 años, dejando de lado participaciones políticas alternativas (Grupo de Memoria Histórica, 2013). La exclusión de participación política y el abandono estatal a las zonas rurales impulsó la conformación de grupos guerrilleros en la década de los sesenta, asentados en el área rural remota y financiados por sus pobladores, gozando de cierta aceptación en sus primeras décadas. A pesar de esto, su expansión en el territorio fue casi nula, lo cual cambio con la intrusión del narcotráfico en la década de los ochenta, generando la expansión de los cultivos de coca y el surgimiento de grupos paramilitares. El cultivo de coca generó cambios en las dinámicas sociales y económicas del conflicto armado y de los habitantes de las zonas rurales remotas dónde fueron sembrados (Gobernación de Caldas \& Oficina de las Naciones Unidas contra la Droga y el Delito, 2019; Narváez-Medina, 2018). Para 1998, el conflicto armado estaba ubicado en zonas específicas del territorio colombiano, situación que cambió drásticamente para 2002 cuando su expansión geográfica fue notable impulsada principalmente por el proceso de paz fallido del gobierno de Andrés Pastrana (1998-2002) con las guerrillas y la ofensiva de los paramilitares que buscaban su fracaso (Grupo de Memoria Histórica, 2013; Salazar, 2016). Luego, con la implementación de la seguridad democrática del gobierno de Álvaro Uribe (2002-2010), el conflicto armado llegó a un pico de incidencia en el territorio nacional acompañado por el actuar de la fuerza pública y la respuesta de las guerrillas. La última etapa del conflicto armado está enmarcada por los diálogos de paz entre las FARC-EP y el gobierno nacional (Juan Manuel Santos 2010-2018), periodo dónde aún se concentraban unos pocos focos de alta incidencia del conflicto armado y que culminó con la firma del tratado de paz en 2016 (Presidencia de la República \& FARC-EP, 2016). El fin del conflicto armado da paso al inicio del postconflicto, periodo dónde se espera consolidar la paz e implementar los acuerdos firmados.

\subsection{Caldas y el postconflicto}

Caldas es un departamento ubicado en el centro occidente de Colombia (ver figura 1) con una población de 998,255 personas y 27 municipios. Su capital Manizales forma junto a Villamaría una zona conurbada donde vive el $46.19 \%$ de los habitantes del departamento (DANE, 2018a). La incidencia del conflicto armado en Caldas vino acompañada por el reemplazo de los cultivos tradicionales por el cultivo de coca a principios de los años noventa en la zona oriente (Samaná, Norcasia y Pensilvania), presionados por grupos armados ilegales provenientes del oriente antioqueño (Gobernación de Caldas \& Oficina de las Naciones Unidas contra la Droga y el Delito, 2019; Narváez-Medina, 2018; Salazar, 2016). Empezando el nuevo milenio, aumentó la presencia de grupos paramilitares en la zona, iniciándose un enfrentamiento entre guerrilla, paramilitares y ejercito que dejó marcas indelebles entre los pobladores de está zona del departamento a través de múltiples asesinatos selectivos, masacres, secuestros y tomas a centros poblados (Grupo de Memoria Histórica, 2013).

El conflicto armado en Colombia ha sido medido a través de distintos índices. En 2014 el Centro de recursos para el Análisis de Conflictos (CERAC) categorizó los municipios de Colombia según la presencia e intensidad del conflicto armado en los últimos 8 años, definiendo Samaná y Pensilvania cómo "fuertemente afectados e interrumpidos" (Schöb, 2014). Así mismo, el Departamento Nacional de Planeación (DNP) en 2016 definió el 
Índice de Incidencia del Conflicto Armado (IICA) (desde Bajo hasta Muy Alto) por municipio según datos de seis variables (Acciones armadas, homicidios, secuestros, minas antipersona, cultivos de coca y desplazamiento forzado). Según este, Caldas cómo departamento posee un IICA Medio Bajo, sin embargo, a nivel de municipios Samaná y Marulanda registran categorías de IICA Muy Alto y Alto (ver figura 1) (Departamento Nacional De Planeación, 2016). Por último, Salazar (2016) analizó datos georreferenciados desde 1986 a 2015, definiendo Caldas cómo una región de afectación alta reciente (2002-2012) por el conflicto armado.

A pesar de la alta incidencia reciente que registran municipios cómo Samaná, Pensilvania y Marulanda, ninguna zona de Caldas fue priorizada en el Programa de Desarrollo con Enfoque Territorial (PDET) el cual busca la transformación de 170 municipios en su ámbito rural históricamente afectados por el conflicto armado, presencia de cultivos ilícitos, pobreza y abandono estatal en el marco del postconflicto (Ministerio de Agricultura y Desarrollo Rural, 2017).

Por este motivo es de suma importancia definir estrategias con enfoque territorial para priorizar la inversión gubernamental que ayudará a cerrar la brecha de las zonas rurales del departamento de Caldas más afectadas por el conflicto armado y el abandono estatal, para así consolidar la paz en sus territorios.

En esta investigación se calculará la accesibilidad geográfica del departamento de Caldas a través de los tiempos de viaje definidos en una matriz origen-destino alimentada con velocidades operacionales extraídas a partir de la DistanceMatrix API de Google (Lahoorpoor \& Levinson, 2020). Luego, se lleva a cabo un modelo geoestadístico para la construcción de las curvas isócronas de cobertura y se relacionan con la población de cada municipio para calcular la accesibilidad geográfica ponderada según la zona urbana y rural, la cual será comparada con el IICA de cada municipio, con el objetivo de buscar la correlación entre las variables (Cardona, Escobar, \& Moncada, 2018).

La accesibilidad es un modelo de transporte que mide las oportunidades de interacción que poseen los individuos (trabajo, educación, salud, recreación) de una región a través de la oferta del transporte (infraestructura vial, modos de transporte) (Aǹez, De La Barra, \& Pérez, 1996; Hansen, 1959). Desde su definición matemática en 1959, investigadores y planificadores de ciudad han desarrollado distintas medidas (Wu \& Levinson, 2020), entre los que se encuentran el modelo de gravedad (Hansen, 1959; Vadrevu \& Kanjilal, 2016), modelo de oportunidades acumuladas (Xi, Miller, \& Saxe, 2018) y la accesibilidad geográfica (Cardona et al., 2018; Walsh, Cullinan, \& Flannery, 2017) cómo los más comunes dada su fácil interpretabilidad. Así mismo, a medida que las capacidades computacionales crecían junto al uso de Sistemas de Información Geográfica (SIG), más variables se han incluido en las medidas dándole más perspectivas a la accesibilidad cómo el ingreso socio-económico de los habitantes sentando las bases para análisis de equidad en el acceso (Jaramillo, Philips, \& Lucas, 2019; Lucas, van Wee, \& Maat, 2016), el género (Melis, Tabasso, Stroscia, \& Costa, 2019) o sistemas de transporte (Guzmán, Oviedo, \& Rivera, 2017). En esta investigación se tendrá en cuenta la diferenciación entre zonas rurales y urbanas (Matsumoto, Kashima, Ogawa, \& Takeuchi, 2013) cómo variable de análisis de accesibilidad geográfica en Caldas, zona de estudio dónde antes se han realizado análisis similares sin tener en cuenta las variables antes mencionadas (Muñoz-Espinosa, 2016; Zuluaga \& Escobar, 2017). 
Figura 1

Ubicación de Caldas en Colombia y categoría de IICA por municipios.

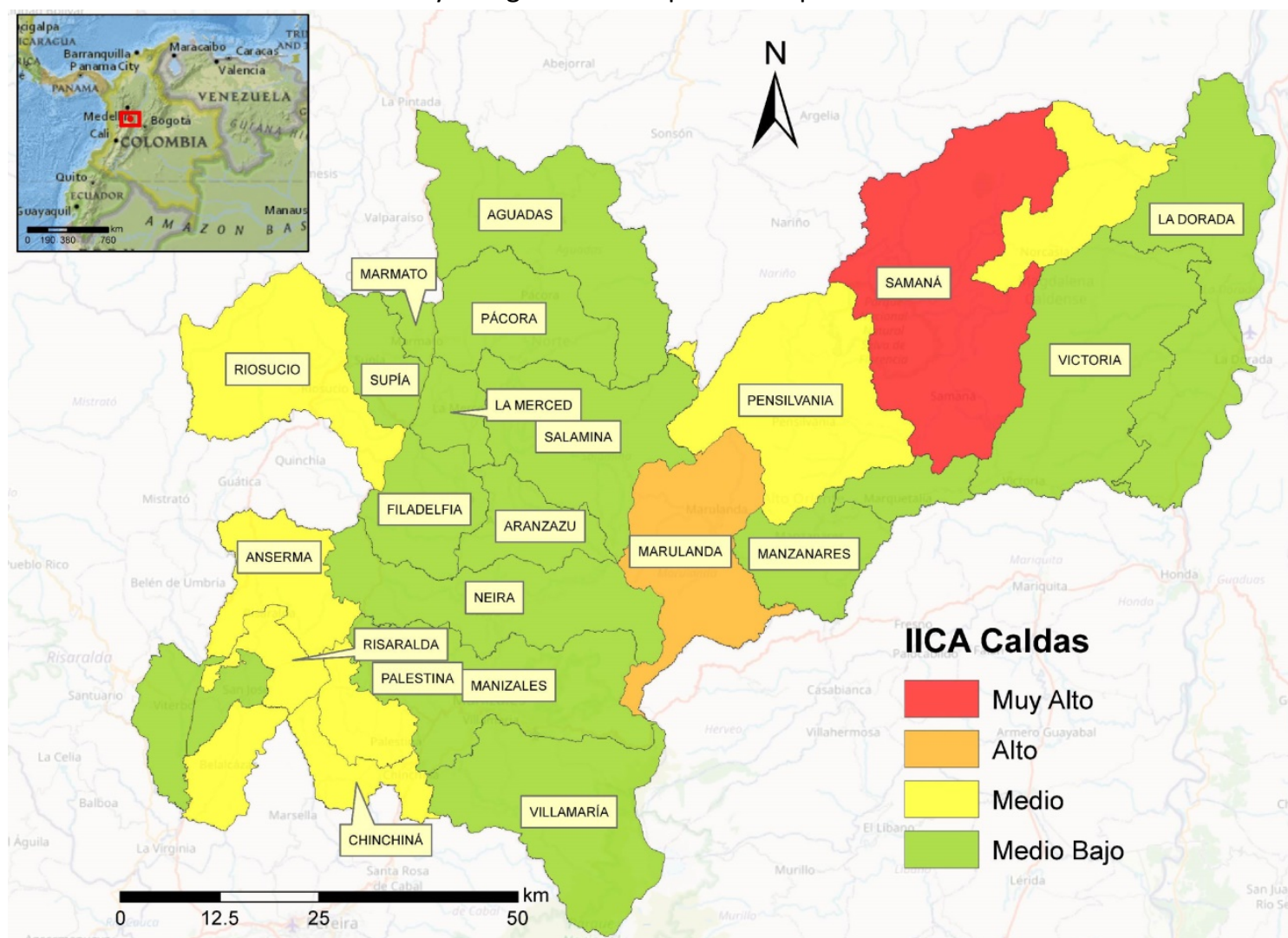

Fuente: Elaboración propia a partir de herramientas de software ArcGis y datos del DNP e IGAC.

A continuación, se presenta la metodología utilizada, explicando detalladamente cada parte del proceso y datos utilizados. Luego, se enumeran los principales resultados y se discuten. Por último, se presentan las conclusiones del estudio.

\section{Metodología}

La metodología de investigación descrita a continuación sigue las etapas mostradas en la figura 2.

\subsection{Recolección de datos}

La primera etapa estaba relacionada con la recolección de datos de entrada para la investigación, enteramente soportada sobre información primaria. En primer lugar, se descargó la división geopolítica y administrativa del departamento a través del Marco Geoestadístico Nacional (MDN) del Departamento Administrativo Nacional de Estadística (DANE), el cual permite la descarga de capas de polígonos georreferenciados de cada municipio categorizados por la clase (urbano y rural) y detallados según el área censal (DANE, 2018b). En segundo lugar, a partir del portal de datos abiertos de la subdirección de cartografía y geográfica del Instituto Geográfico Agustín Codazzi (IGAC) se obtuvo la red vial departamental georreferenciada (IGAC, 2020). En tercer lugar, el IICA por municipio se descargó del DNP en formato CSV (Departamento Nacional De Planeación, 2016). Por último, los datos del Censo Nacional de Población y Vivienda de 2018 (CNPV2018) del DANE se descargaron en formato CSV del área de micro datos de la misma entidad (DANE, 2018a). 


\subsection{Puesta a punto de las capas de información geográfica}

En esta etapa se organizaron las capas necesarias para el cálculo de la accesibilidad geográfica: red de infraestructura vial y división político-administrativa del departamento según el área censal.

Para la puesta a punto de la red de infraestructura vial se tuvieron en cuenta dos apreciaciones básicas. La primera está relacionada con la teoría de grafos, la cual indica que para modelos de transportes las vías equivalen a arcos y las intersecciones a nodos (Schultes, 2008; Weber, 2016). Además, para los cálculos de accesibilidad geográfica y modelos geoestadísticos es necesaria la maximización de datos en el territorio espacial con el objetivo de aumentar su correlación y precisión predictiva por lo que los arcos de la red de infraestructura vial se dividieron hasta dejarlos de máximo 300 m usando la librería Geopandas y Shapely de Python 3.7 (Gillies, \& others, 2007; Jordahl, 2014). Por otro lado, las velocidades de operación fueron introducidas en la red a partir de rutas entre ciudades calculadas a partir de la DistanceMatrix Api de Google. A está se le realiza un requerimiento usando coordenadas geográficas de dos puntos conocidos, entregando la distancia y tiempo de viaje, con la que se calcularon las velocidades y se introdujeron cómo variables en los arcos correspondientes.

Por otro lado, la puesta a punto de la división político-administrativa del departamento según el área censal se llevó a cabo relacionando sus códigos únicos con los del CNPV2018, teniendo en cuenta la población rural y urbana y distribuyendo según el área del polígono. Para ello se utilizaron las librerías Geopandas y Pandas de Python 3.7 (Jordahl, 2014; Mckinney, 2017).

Figura 2

Metodología de investigación

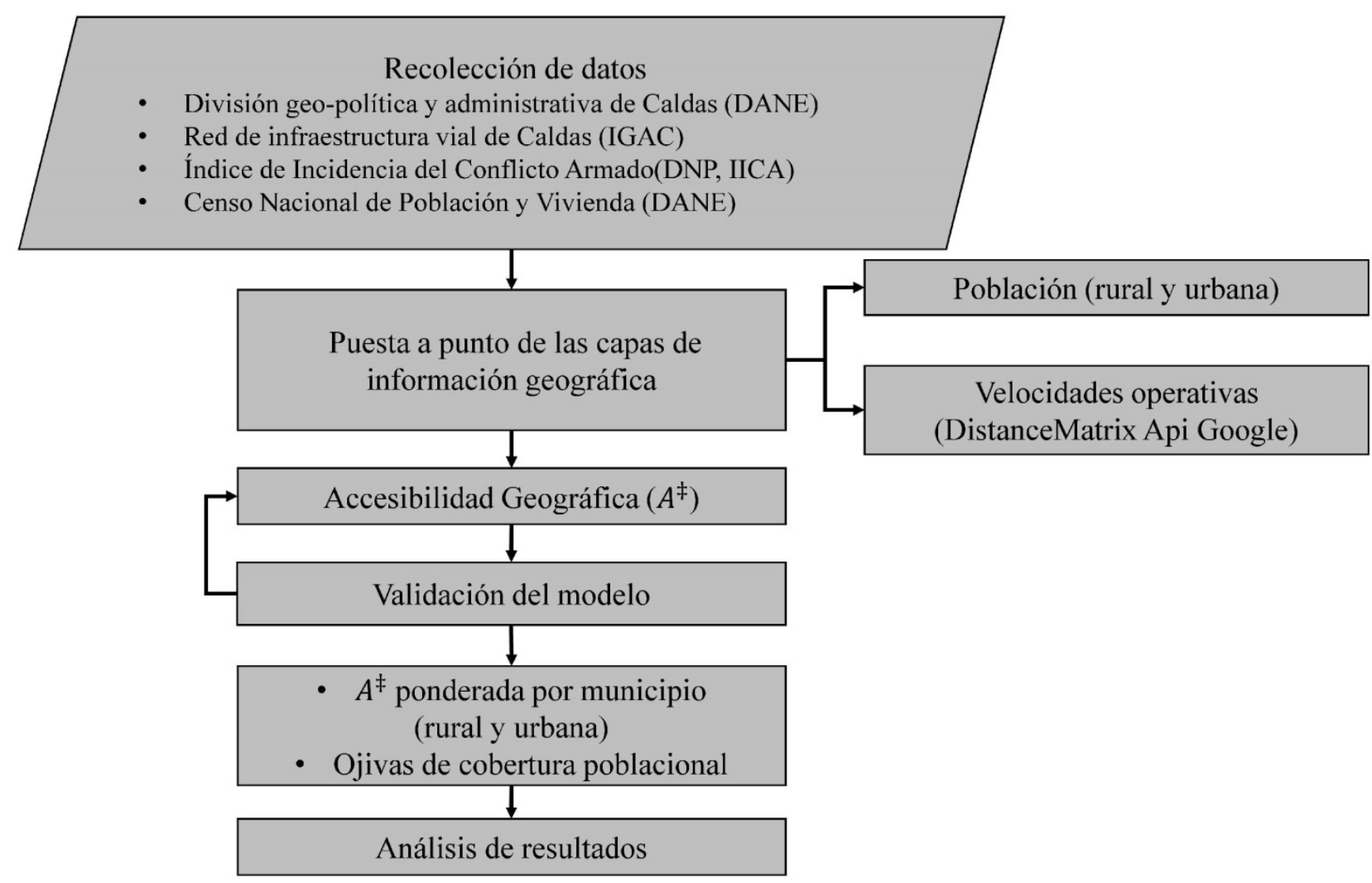

Fuente: Elaboración propia 


\subsection{Accesibilidad geográfica}

Para el cálculo de la accesibilidad geográfica $\left(\boldsymbol{A}^{\ddagger}\right)$ se calcula la impedancia de viaje $\left(\boldsymbol{C}_{\boldsymbol{i} \boldsymbol{j}}\right)$ entre todos los nodos de la red de infraestructura vial cómo origen (i) y destino (j) de los mismos, usando el camino mínimo según el algoritmo de Dijsktra (Dijkstra, 1959), la red de infraestructura vial y el software Transcad 8.0, luego de haber pasado el proceso de validación (Wu \& Levinson, 2020).

$$
A^{\ddagger}=\frac{\sum_{i=1}^{J} \sum_{j=1}^{J} C_{i j}}{J-1}
$$

Luego de calcular la accesibilidad geográfica cómo el tiempo de viaje promedio de cada nodo de la red vial hacia todos los demás se construyeron las curvas isócronas mediante el método geoestadístico Kriging ordinario con semivariograma lineal cómo ecuación estructurante, el cual predice los tiempos de viaje a partir de la correlación espacial y la distancia entre los valores, en este caso el tiempo de viaje. Este modelo ha demostrado ser el interpolador óptimo, dados los buenos resultados obtenidos en diversas investigaciones relacionadas con transporte (Cardona et al., 2018; Gomes, Pitombo, Rocha, \& Salgueiro, 2016).

\subsection{Calibración y validación del modelo}

El proceso de calibración y validación de modelo fue realizado comparando los tiempos de viaje mínimos entre los centros poblados de los 27 municipios del departamento usando la DistanceMatrixApi de Google con los resultados dados por el algoritmo de caminos mínimos de Transcad 8.0, usando la red vial ajustada en la segunda etapa (Lahoorpoor \& Levinson, 2020). En el caso de que los resultados del modelo fueron menores a los de la realidad se usaron las penalidades por giro cómo variable de calibración (Cardona, Escobar, \& Moncada, 2019; Yiannakoulias, Bland, \& Svenson, 2013). Finalmente, se usó una regresión lineal entre los datos cómo método de validación, buscando un coeficiente de regresión mayor al 95\% y una correlación de Pearson cercana a 1.

\subsection{Accesibilidad geográfica ponderada y ojivas de cobertura}

En esta etapa se relacionaron las curvas isócronas de accesibilidad geográfica con la división políticoadministrativa del departamento en su área censal, calculando las ojivas de cobertura de población total y según la clase: urbano y rural. También, se calculó la accesibilidad geográfica ponderada por municipio $\left(\overline{\boldsymbol{A}}_{\boldsymbol{m}}^{\ddagger}\right)$ según la clase de su territorio (urbano y rural) a partir de la ecuación 2 dónde C corresponde al tiempo promedio de viaje de la isócrona de accesibilidad n y p la población en la misma (Escobar, Cardona, \& Moncada, 2020).

$$
{\overline{A^{\ddagger}}}_{m}=\frac{p_{1} * \overline{C_{1}}+p_{2} * \overline{C_{2}}+\cdots+p_{n} * \overline{C_{n}}}{p_{1}+p_{2}+\cdots+p_{n}}(2)
$$

\subsection{Análisis de resultados}

En esta etapa se realizarán análisis cuantitativos entre las variables estudiadas con el objetivo de encontrar significancia estadística y correlación espacial que permita determinar la forma cómo se relaciona la accesibilidad geográfica y el conflicto armado.

\section{Resultados}

\subsection{Red de infraestructura vial y área censal departamento de Caldas}

En primer lugar, se presentan los resultados de la puesta a punto de la red vial y del polígono de área censal de Caldas en la figura 3. El resumen de las variables descriptivas de la red vial departamental se presenta en la tabla 1. La red departamental está compuesta por 16,222 nodos, 16,274 arcos y 2,263 kilómetros de vías, siendo el 
intervalo de velocidad entre 20 y $30 \mathrm{~km} / \mathrm{h}$ el que mayor $\mathrm{km}$ poseen (43.31\%). Por otro lado, se presenta el área censal, dónde el 74.35\% de los habitantes se encuentran en zonas urbanas, principalmente en la conurbación de la capital Manizales con Villamaría.

Figura 3

Red vial departamental, áreas censales y división político-administrativa de Caldas.

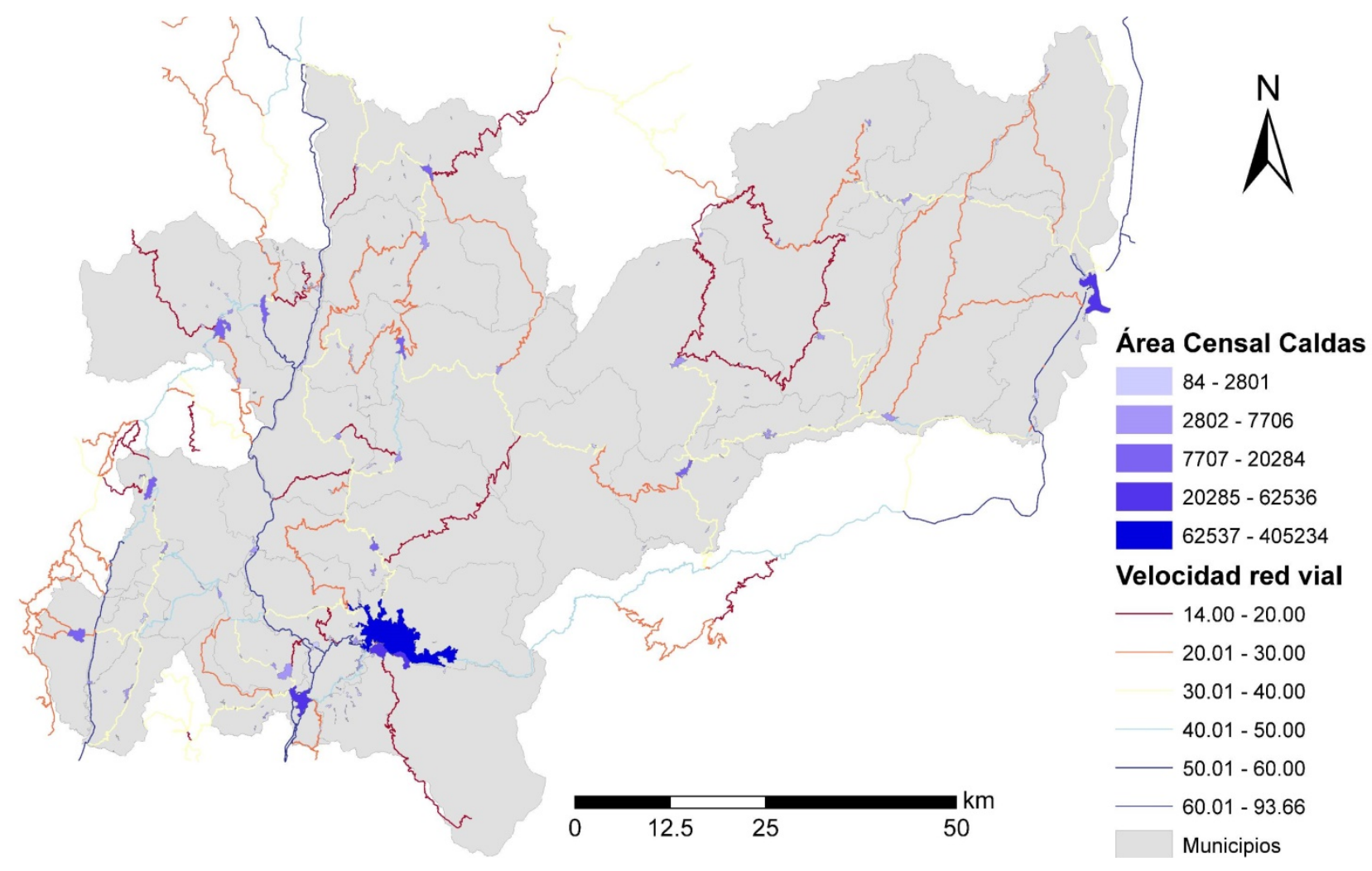

Fuente: Elaboración propia en ArcGis a partir de datos del DANE e IGAC

Por otro lado, en la figura 4 se presenta la validación del modelo de accesibilidad geográfica del departamento de Caldas mediante la comparación de los tiempos de viaje entre los centros poblados calculados con la herramienta de caminos mínimos de Trasncad 8.0 y los tiempos de viaje calculados a través de la DistanceMatrix API de Google. En total se validaron 749 rutas correspondientes a los centros poblados de los 27 municipios del departamento cómo origen y destino de los viajes. En el proceso de calibración y modelación se definieron unas penalidades por giro de $0.75 \mathrm{~min}$ a la izquierda y $0.5 \mathrm{~min}$ a la derecha. Además, en la tabla 3 se presentan algunos indicadores de la validación del modelo, con un coeficiente de Pearson cercano a 1, un coeficiente de regresión del $97 \%$ y un p-value menor a 0.001 , lo que nos muestra que la red de infraestructura vial representa de buena manera la realidad en cuanto a la movilidad del departamento. 
Tabla 1

Variables descriptivas de

la red vial departamental.

\begin{tabular}{|c|c|}
\hline Número de nodos & 16222 \\
\hline Número de Arcos & 16274 \\
\hline Velocidad $(\mathrm{km} / \mathrm{h})$ & Longitud $(\mathrm{km})$ \\
\hline$<20$ & 293 \\
\hline$[20-30)$ & 980 \\
\hline$[30-40)$ & 765 \\
\hline$[40-50)$ & 316 \\
\hline$[50-60)$ & 158 \\
\hline$>=60$ & 151 \\
\hline Total & 2263 \\
\hline Fuente: Elaboracion & \\
\hline
\end{tabular}

Fuente: Elaboración propia

Figura 4

Validación tiempos de viaje mínimos calculados en Transcad 8.0 y Distancematrix API de Google.

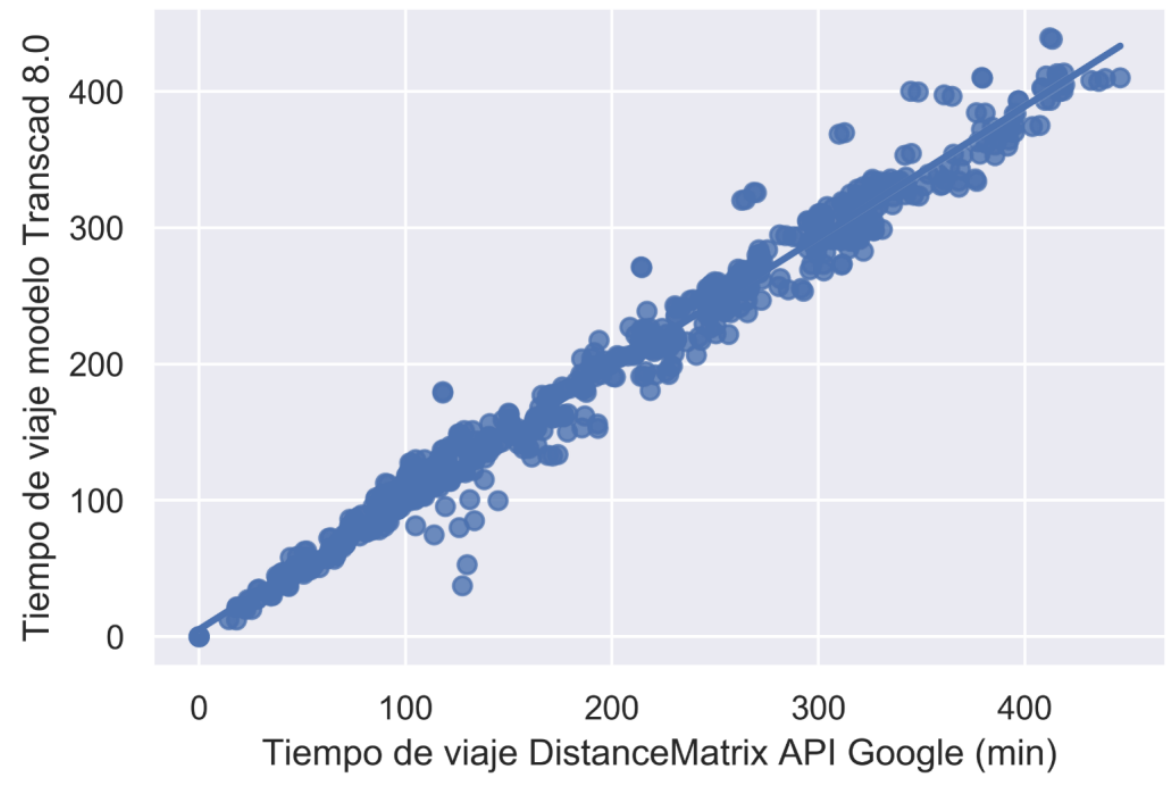

Fuente: Elaboración propia

Tabla 2

Indicadores de la validación

\begin{tabular}{|c|c|}
\hline Correlación de Pearson & 0.9901 \\
\hline $\boldsymbol{p}$-value & $<0.001$ \\
\hline $\mathbf{R}^{\wedge} \mathbf{2}$ & 0.9795 \\
\hline
\end{tabular}

Fuente: Elaboración propia 


\subsection{Accesibilidad Geográfica}

En la figura 5 se aprecia la accesibilidad geográfica para el departamento de Caldas, resultando en 167 minutos cómo tiempo promedio de viaje mínimo, en la zona de Manizales, la capital y 343 minutos cómo tiempo de viaje máximo en zonas al noreste del departamento, dónde se ubican municipios cómo Norcasia y Samaná. Los círculos azules enmarcan las zonas del departamento con mejor accesibilidad geográfica, relacionadas con las vías que conectan la capital (Manizales) con las dos ciudades más importantes del país, Medellín al norte y Bogotá al este. Por ello, los municipios alrededor de estás vías poseen una accesibilidad geográfica adecuada, beneficiados por el desarrollo de infraestructura necesario para conectar la capital con nodos importantes de la economía nacional (Muñoz-Espinosa, 2016; Zuluaga \& Escobar, 2017). Por otro lado, en circulo rojo se encuentran las zonas con mayores tiempos promedio de viaje. Estos zonas han sido afectadas ampliamente por el conflicto armado (Samaná, Pensilvania y Norcasia), específicamente el norte de Samaná y Norcasia dónde históricamente se concentraron los cultivos de coca (Gobernación de Caldas \& Oficina de las Naciones Unidas contra la Droga y el Delito, 2019; Narváez-Medina, 2018).

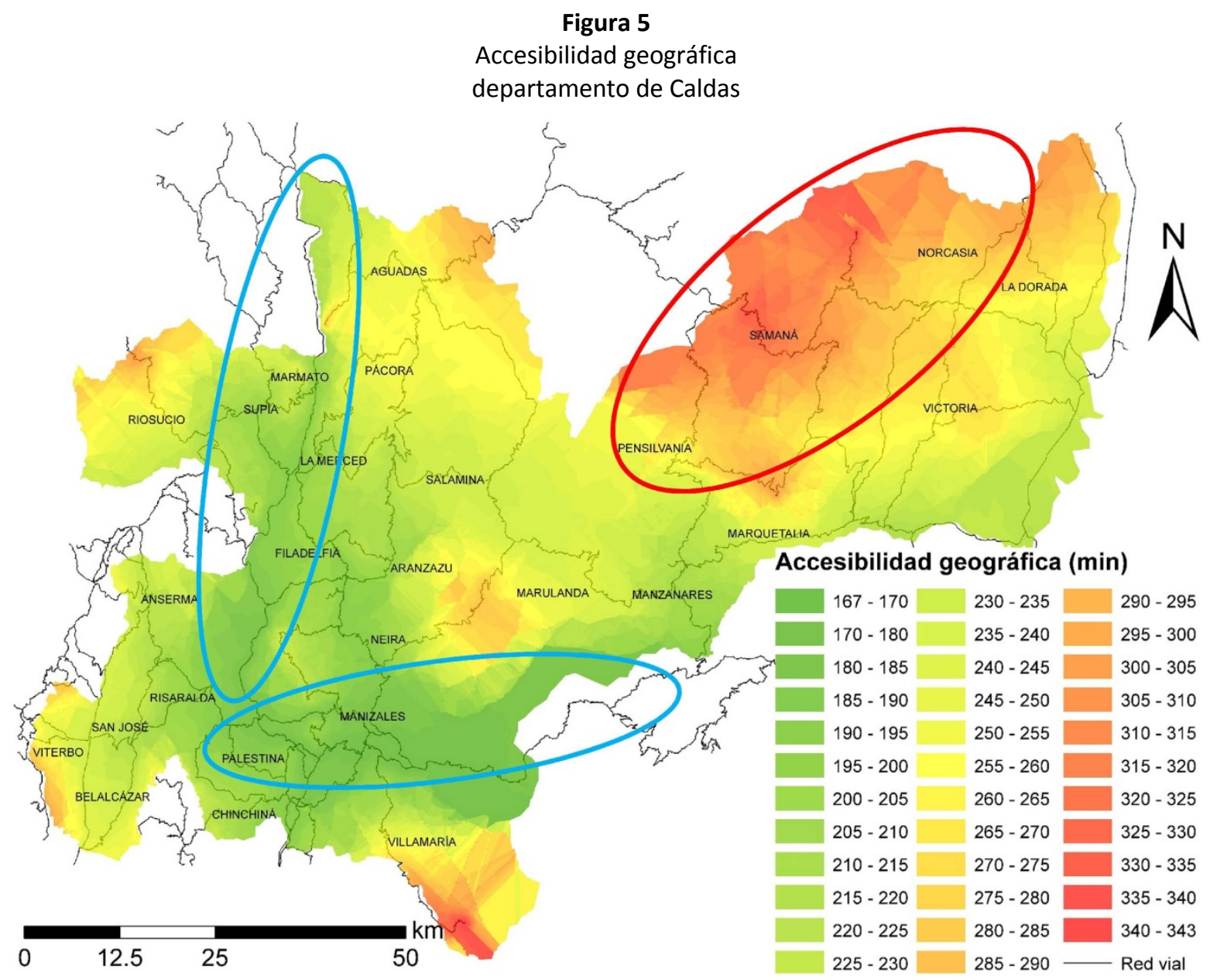

Fuente: Elaboración propia.

En la figura 6 se presenta la ojiva de porcentaje acumulada de población según el tiempo de viaje, dónde el 80\% de la población del departamento están cubiertos en tiempos promedio de viaje de 235 minutos o menos. Por otra parte, la figura 7 presenta la ojiva de porcentaje acumulado de población según su clase, urbano o rural. Allí se puede apreciar una clara disparidad entre la zona urbana y rural dado que para el $80 \%$ de población en la zona urbana percibe tiempos promedio de viaje de 230 min o menos mientras que para la zona rural el tiempo promedio de viaje es de $250 \mathrm{~min}$. La diferencia entre el acceso de la zona rural y urbana debe ser atendida por 
los gobernantes, buscando satisfacer las necesidades básicas de las zonas remotas (Shalini, 2015), especialmente para que el conflicto armado no retorne a estos lugares.

Figura 6

Ojiva porcentual de

población acumulada.

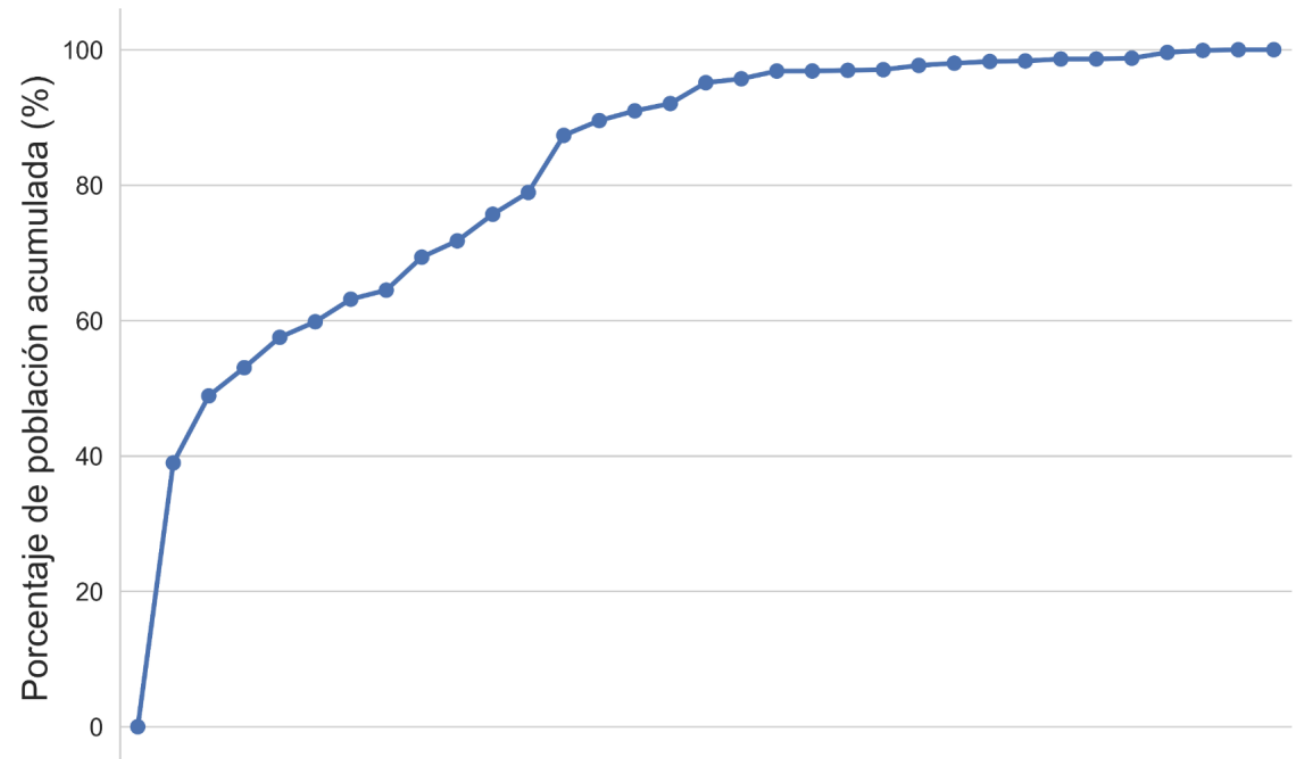

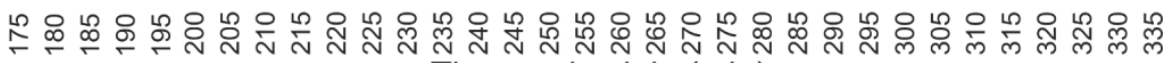

Tiempo de viaje (min)

Fuente: Elaboración propia

Figura 7

Ojiva porcentual de población acumulada: zona urbana y rural.

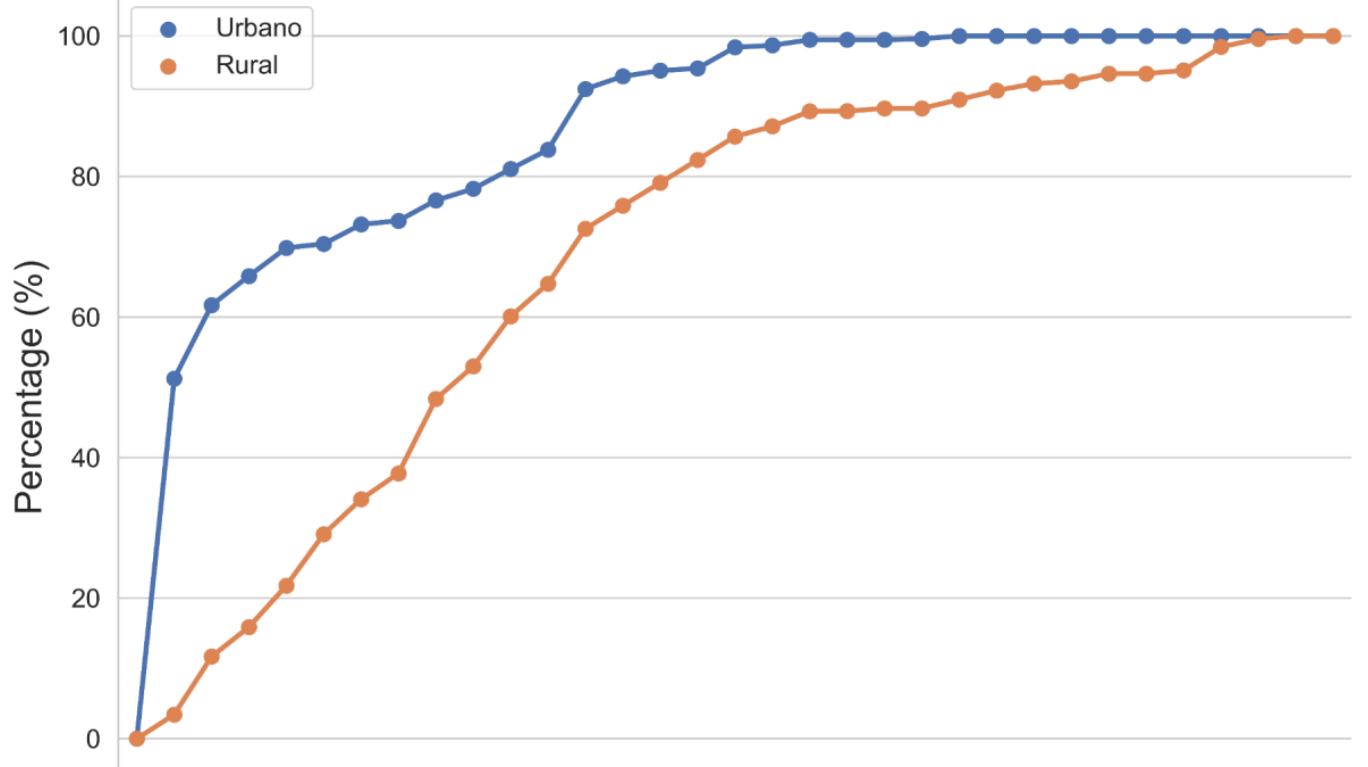

얃 \& Tiempo de viaje (min)

Fuente: Elaboración propia 
Por ello, la cobertura poblacional a través de la red de infraestructura vial resulta fundamental para aumentar las posibilidades de interacción de las zonas rurales, en temas importantes cómo el comercio de cultivos, salud y educación superior (Cullinan, Flannery, Walsh, \& McCoy, 2013; Matsumoto et al., 2013).

Los resultados de accesibilidad geográfica ponderada por municipio, según la zona urbana y rural se presentan en la tabla 3. Allí se aprecia que Manizales posee el mejor indicador con 178.30 min para la zona urbana y 183.06 min para la zona rural. Por otro lado, Norcasia es el municipio el peor indicador de accesibilidad geográfica ponderada para la zona urbana con $\mathbf{2 8 1 . 2 9}$ min mientras que Samaná tiene el peor indicador en la zona rural con $309.40 \mathrm{~min}$.

Tabla 3

Accesibilidad geográfica ponderada por municipio, zona urbana y rural.

\begin{tabular}{|c|c|c|c|c|}
\hline Municipio & Urbano & Rural & \multicolumn{2}{|c|}{ IICA } \\
\hline Aguadas & 253.25 & 252.47 & 0.0171565 & Medio Bajo \\
\hline Anserma & 226.81 & 227.35 & 0.0223358 & Medio \\
\hline Aránzazu & 205.24 & 202.24 & 0.0107323 & Medio Bajo \\
\hline Belalcázar & 232.64 & 235.10 & 0.0195218 & Medio \\
\hline Chinchiná & 189.38 & 204.62 & 0.0234982 & Medio \\
\hline Filadelfia & 202.50 & 205.98 & 0.0150543 & Medio Bajo \\
\hline La Dorada & 232.55 & 251.14 & 0.0104393 & Medio Bajo \\
\hline La Merced & 218.87 & 205.02 & 0.0117015 & Medio Bajo \\
\hline Manizales & 178.30 & 183.06 & 0.0089991 & Medio Bajo \\
\hline Manzanares & 212.50 & 211.93 & 0.0134387 & Medio Bajo \\
\hline Marmato & 220.00 & 217.87 & 0.0119945 & Medio Bajo \\
\hline Marquetalia & 235.69 & 242.50 & 0.0141166 & Medio Bajo \\
\hline Marulanda & 242.50 & 182.50 & 0.0552795 & Alto \\
\hline Neira & 192.37 & 188.72 & 0.012848 & Medio Bajo \\
\hline Norcasia & 281.29 & 284.93 & 0.0297625 & Medio \\
\hline Pácora & 252.71 & 250.66 & 0.0123045 & Medio Bajo \\
\hline Palestina & 197.15 & 194.07 & 0.0195184 & Medio \\
\hline Pensilvania & 250.93 & 279.53 & 0.0252295 & Medio \\
\hline Riosucio & 215.84 & 217.16 & 0.0209369 & Medio \\
\hline Risaralda & 212.52 & 202.66 & 0.0199034 & Medio \\
\hline Salamina & 222.98 & 231.76 & 0.0149483 & Medio Bajo \\
\hline Samaná & 262.50 & 309.40 & 0.0827464 & Muy Alto \\
\hline San José & 222.43 & 237.23 & 0.0119994 & Medio Bajo \\
\hline Supía & 202.55 & 206.03 & 0.0154873 & Medio Bajo \\
\hline Victoria & 222.26 & 253.71 & 0.0122667 & Medio Bajo \\
\hline Villamaría & 179.42 & 196.52 & 0.0065088 & Medio Bajo \\
\hline Viterbo & 239.05 & $\mathrm{NaN}$ & 0.0188785 & Medio Bajo \\
\hline
\end{tabular}

Fuente: Elaboración propia

Además, la tabla 3 presenta los valores de IICA por cada municipio, los cuales fueron evaluados junto a la accesibilidad geográfica urbana y rural encontrando una relación directa (ver tabla 4) dado que a medida que aumenta la accesibilidad geográfica ponderada, aumenta la incidencia del conflicto armado en el municipio (ver figura 8), lo que muestra el rezago en infraestructura derivado por la presencia del conflicto armado y la importancia de su mejoramiento en la construcción de paz. 
Taba 4

Indicadores de relación IICA y Accesibilidad geográfica ponderada

\begin{tabular}{|c|c|c|}
\cline { 2 - 3 } \multicolumn{1}{c|}{} & \multicolumn{2}{c|}{ IICA } \\
\cline { 2 - 3 } \multicolumn{1}{c|}{} & CC & $\boldsymbol{p}$-value \\
\hline Urbano & 0.5006 & 0.0091883 \\
\hline Rural & 0.4178 & 0.0336726 \\
\hline \multicolumn{2}{|c|}{ CC $=$ Correlación Pearson } \\
\hline
\end{tabular}

Fuente: Elaboración propia

Figura 8

Diagramas de correlación entre el IICA y la accesibilidad geográfica ponderada: urbana y rural.
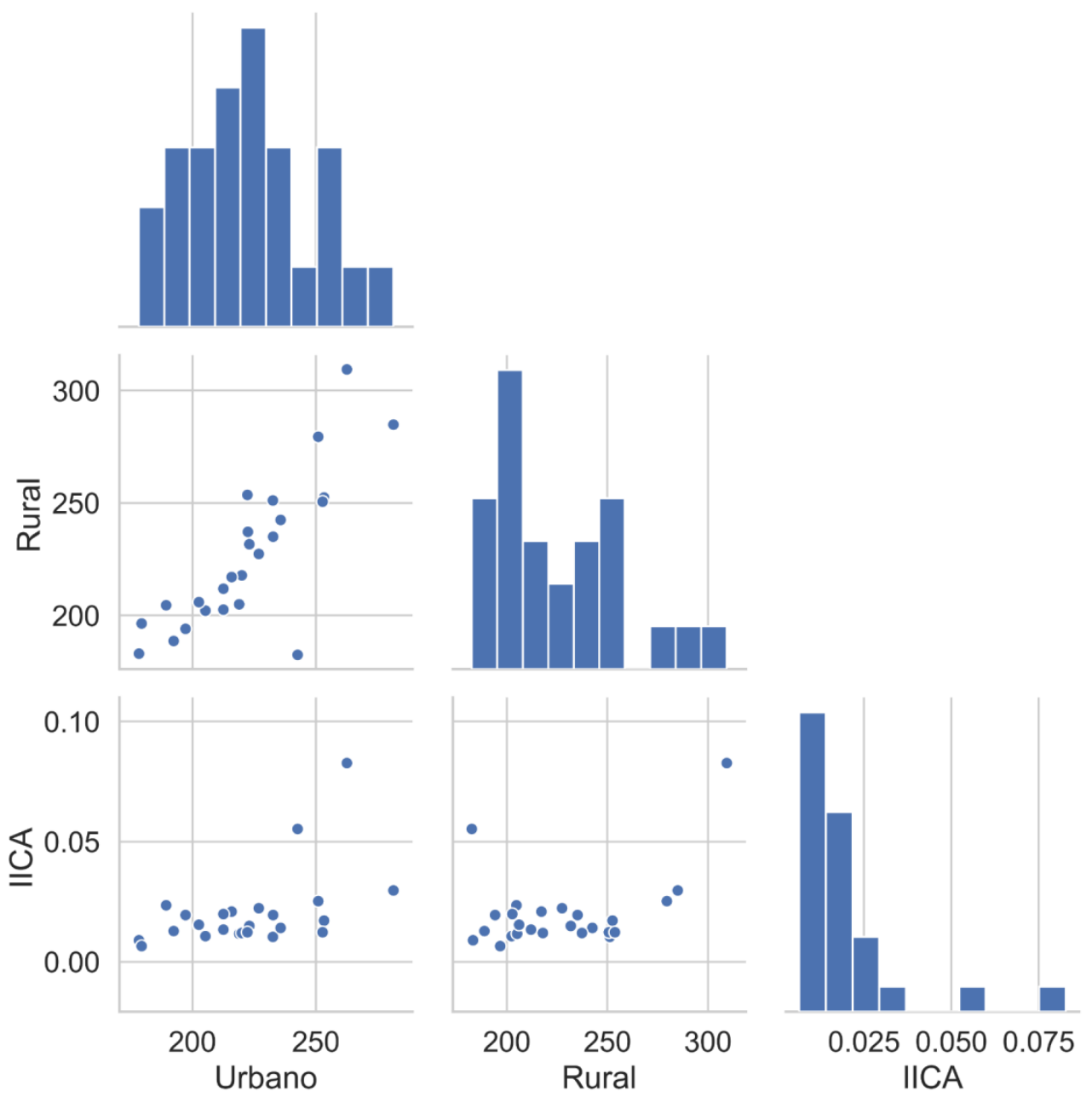

Fuente: Elaboración propia

\section{Conclusiones}

Este artículo de investigación muestra una metodología replicable y de amplia interpretabilidad para los tomadores de decisiones cómo lo es la accesibilidad geográfica. Así mismo, por primera vez, se usan los servicios de Google Maps para validar la matriz de tiempos de viaje calculada en Transcad 8.0, la cual depende de 
velocidades funcionales u operacionales extraídas de distintas fuentes de información. Esto permite dar un paso adelante, yaqué el cálculo de los tiempos de viaje es un paso crucial en el cálculo de la accesibilidad geográfica y de otros modelos de transporte.

La accesibilidad geográfica ponderada es un indicador importante acerca de las posibilidades de interacción en una región, así como de la calidad de su red de infraestructura vial. Este puede ser combinado con otros indicadores con el objetivo de evaluar proyectos de infraestructura vial y el impacto en la región.

Las velocidades operacionales de la red de infraestructura vial son bajas respecto a otras zonas geográficas dadas las condiciones topográficas del departamento, haciendo difícil y costoso el mejoramiento y/o construcción de vías. Esto hace aún más importante la evaluación de proyectos para la priorización de la inversión estatal.

Teniendo en cuenta la relación entre la accesibilidad geográfica ponderada y el IICA, la investigación revela las zonas del departamento donde debe mejorarse la infraestructura vial con el objetivo de mejorar el potencial de interacción de sus habitantes y así evitar que el conflicto armado retorne. Por esto, la zona norte de Samaná y Norcasia deben tener especial atención en el futuro en cuanto a inversión en infraestructura vial para que su conexión con el resto del departamento aumente. Por otra parte, es clara la diferencia entre los indicadores de accesibilidad geográfica para los habitantes de la zona rural y urbana, lo que genera que las brechas de desigualdad se acentúen.

Relacionado con el conflicto armado y la etapa del postconflicto en Colombia, Caldas debería ser incluida entre los municipios PDET, dado que zonas cómo Samaná y Pensilvania resultaron ampliamente afectados, según los distintos índices calculados por instituciones gubernamentales y privadas, lo que les permitirá crear un Plan Integral de Desarrollo que evite el retorno del conflicto a su región.

En cuanto a investigaciones futuras, está metodología puede ser aplicada en todo Colombia para establecer la relación entre el conflicto armado y el desarrollo de la infraestructura vial, buscando priorizar la inversión en las zonas afectadas por el conflicto armado. También, puede ser utilizada en otros países y contextos para la evaluación de proyectos de infraestructura dada su fácil replicabilidad en cuanto a tiempo y dinero.

\section{Agradecimientos}

Los investigadores agradecen al Ministerio de Ciencia, Tecnología e Innovación de Colombia y a la Universidad Nacional de Colombia sede Manizales por el apoyo a través de la Convocatoria 812 de 2018 "Jóvenes Investigadores e Innovadores". Así mismo, al Grupo de Investigación en Movilidad Sostenible (GIMS). Este artículo de investigación "Full Paper" ha sido sometido individualmente a revisión de pares académicos.

\section{Referencias bibliográficas}

Aǹez, J., De La Barra, T., \& Pérez, B. (1996). Dual graph representation of transport networks. Transportation Research Part B: Methodological, 30(3), 209-216. https://doi.org/10.1016/0191-2615(95)00024-0

Calderón Rojas, J. (2016). Etapas del conflicto armado en Colombia: hacia el postconflicto. Revista de Estudios Latinoamericanos, 1(62), 227-257.

Cardona, S., Escobar, D. A., \& Moncada, C. A. (2018). Análisis de Accesibilidad como Herramienta para la Generación de Zonas Libres de Tráfico. Caso del Municipio de La Dorada en Colombia. Información Tecnológica, 29(5), 203-214. https://doi.org/10.4067/S0718-07642018000500203

Cardona, S., Escobar, D. A., \& Moncada, C. A. (2019). Cálculo de las penalidades por giro en modelos de transporte acorde al tipo de vehículo. Caso de estudio: Manizales, Caldas. Revista Espacios, 40(38), 22-36. 
Cullinan, J., Flannery, D., Walsh, S., \& McCoy, S. (2013). Distance effects, social class and the decision to participate in higher education in Ireland. Economic and Social Review, 44(1), 19-51.

Departamento Administrativo Nacional de Estadística-DANE (2018a). Colombia -Censo Nacional de Población y Vivienda - CNPV - 2018. Recuperado de:

http://microdatos.dane.gov.co/index.php/catalog/643/get_microdata

Departamento Administrativo Nacional de Estadística-DANE (2018b). Manual de uso del Marco Geoestadístico Nacional en el proceso estadístico. Recuperado de: https://geoportal.dane.gov.co/servicios/descarga-ymetadatos/descarga-mgn-marco-geoestadistico-nacional/

Departamento Nacional De Planeación. (2016). Índice de Incidencia del Conflicto Armado en Colombia (IICA).

Dijkstra, E. W. (1959). A note on two problems in connexion with graphs. Numerische Mathematik, 1(1), 269271.

Escobar D. A., Cardona S, Moncada C. A. Evaluación de Infraestructura Mediante Accesibilidad Territorial. Caso de Estudio: Conexión Villamaría-Manizales (Colombia). Inf. Tecnol. 2020;31(3); in press.

Gillies, S., \& others. (2007). Shapely: manipulation and analysis of geometric objects. Recuperado de: https://github.com/Toblerity/Shapely

Gobernación de Caldas, \& Oficina de las Naciones Unidas contra la Droga y el Delito. (2019). Caldas libre de cultivos de coca: logros y desafíos.

Gomes, V. A., Pitombo, C. S., Rocha, S. S., \& Salgueiro, A. R. (2016). Kriging Geostatistical Methods for Travel Mode Choice: A Spatial Data Analysis to Travel Demand Forecasting. Open Journal of Statistics, 6(3), 514527. https://doi.org/10.4236/ojs.2016.63044

Grupo de Memoria Histórica. (2013). ¡Basta Ya! Colombia: Memorias de guerra y dignidad. En Centro Nacional de Memoria Histórica.

Guzmán, L. A., Oviedo, D., \& Rivera, C. (2017). Assessing equity in transport accessibility to work and study: The Bogotá region. Journal of Transport Geography, 58, 236-246.

https://doi.org/10.1016/j.jtrangeo.2016.12.016

Hansen, W. G. (1959). How Accessibility Shapes Land Use. Journal of the American Planning Association, 25(2), 73-76. https://doi.org/10.1080/01944365908978307

Instituto Geográfico Agustín Codazzi - IGAC. (2020). Datos Abiertos Cartografía y Geografía. Recuperado de: https://geoportal.dane.gov.co/servicios/descarga-y-metadatos/descarga-mgn-marco-geoestadisticonacional/

Jaramillo, A. G., Philips, I., \& Lucas, K. (2019). Social impact assessment: The case of bus rapid transit in the City of Quito, Ecuador. In Measuring Transport Equity. https://doi.org/10.1016/B978-0-12-814818-1.00014-7.

Jordahl, K. (2014). GeoPandas: Python tools for geographic data. Recuperado de: Https://Github. Com/Geopandas/Geopandas.

Lahoorpoor, B., \& Levinson, D. M. (2020). The Transit Travel Time Machine: Comparing Three Different Tools for Travel Time Estimation. Transportation Research Board, Washington, DC., 20. Washington DC. 
Lucas, K., van Wee, B., \& Maat, K. (2016). A method to evaluate equitable accessibility: combining ethical theories and accessibility-based approaches. Transportation, 43(3). https://doi.org/10.1007/s11116-0159585-2

Matsumoto, M., Kashima, S., Ogawa, T., \& Takeuchi, K. (2013). Do rural and remote areas really have limited accessibility to health care? Geographic analysis of dialysis patients in Hiroshima, Japan. Rural and Remote Health, 13(3), 2507.

Mckinney, W. (2017). Python for Data Analysis - 2nd edition. Recuperado de: https://doi.org/10.1145/1985441.1985476

Melis, G., Tabasso, M., Stroscia, M., \& Costa, G. (2019). Assessing health inequalities related to urban and transport determinants of mental health. In Measuring Transport Equity. https://doi.org/10.1016/B978-012-814818-1.00009-3

Ministerio de Agricultura y Desarrollo Rural. (2017). Decreto 893 de 2017. Recuperado de: http://es.presidencia.gov.co/normativa/normativa/DECRETO\%20893\%20DEL\%2028\%20DE\%20MAYO\%20 DE\%202017.pdf

Muñoz-Espinosa, A. F. (2016). Análisis de accesibilidad territorial a nivel regional y el impacto de las concesiones 4G Proyecto Autopistas para la Prosperidad Pacifico 1, 2 y 3. Tesis de Maestría. Universidad Nacional de Colombia. Manizales. Recuperado de: http://www.bdigital.unal.edu.co/53789/

Narváez-Medina, D. A. (2018). El conflicto armado como factor de transformación territorial del Oriente de Caldas, Colombia. Civilizar, 18(35), 13-24. https://doi.org/10.22518/usergioa/jour/ccsh/2018.2/a02

Pérez, R., Salazar, A., Foster, W., \& Osses, P. (2013). El efecto de la distancia al mercado sobre la pobreza rural en la Región Metropolitana de Santiago. Eure, 39(116), 173-188. https://doi.org/10.4067/S025071612013000100007

Presidencia de la República, \& FARC-EP. (2016). Acuerdo final para la terminación del conflicto y la construcción de una paz estable y duradera. Recuperado de: http://www.altocomisionadoparalapaz.gov.co/procesos-yconversaciones/Documentos compartidos/24-11-2016NuevoAcuerdoFinal.pdf

Salazar, G. S. (2016). El conflicto armado y su incidencia en la configuración territorial: Reflexiones desde la ciencia geográfica para la consolidación de un periodo de pos-negociación del conflicto armado en Colombia. Bitácora Urbano Territorial, 26(2), 45. https://doi.org/10.15446/bitacora.v26n2.57605

Schöb, M. (2014). Demobilizing and Reintegrating Whom? Accounting for Diversity in DDR Processes: An Analysis of the Colombian case. En Centro de Recursos para el Análisis de Conflictos (CERAC). Retrieved from http://cerac.org.co/assets/pdf/Other publications/WP21_Mia Schöb.pdf

Schultes, D. (2008). Route Planning in Road Networks. En Ausgezeichnete Informatikdissertationen, 271-280.

Shalini, K. (2015). Quantification of Rural Accessibility and Development of a Need-Based Approach for Rural Road Network Planning. Tesis doctoral. BIRLA INSTITUTE OF TECHNOLOGY \& SCIENCE.

Sandino, V., Simanca, F.A., Páez, J.A., Cortéz-Méndez, J., Palacios-Rozo, J.J, \& Alfaro, A.C. (2019). El proceso de reincorporación de las mujeres de las Fuerzas Armadas Revolucionarias de Colombia - Ejército del Pueblo: necesidades, retos y perspectivas. En Meriño, V., Martinez, E., \& Martínez, C. (Ed.). 1". Vol. 14, Año 14, Pág. 335-354. Santa Bárbara - Zulia - Venezuela: Fondo Editorial Universitario de la Universidad Nacional 
Experimental del Sur del Lago de Maracaibo Jesús María Semprún. Recuperado de:

http://www.unesur.edu.ve/libros

Ugarriza, J. E. (2013). La dimensión política del postconflicto: discusiones conceptuales y avances empíricos. Colombia Internacional, (77), 141-176.

Vadrevu, L., \& Kanjilal, B. (2016). Measuring spatial equity and access to maternal health services using enhanced two step floating catchment area method (E2SFCA) - A case study of the Indian Sundarbans. International Journal for Equity in Health, 15(1), 1-12. https://doi.org/10.1186/s12939-016-0376-y

Walsh, S., Cullinan, J., \& Flannery, D. (2017). The Impact of Proposed Higher Education Reforms on Geographic Accessibility to Universities in Ireland. Applied Spatial Analysis and Policy, 10(4), 515-536. https://doi.org/10.1007/s12061-016-9193-3

Weber, J. (2016). The properties of topological network connectivity measures and their application to U.S. urban freeway networks. Professional Geographer, 68(3), 485-495. https://doi.org/10.1080/00330124.2015.1106324

Wu, H., \& Levinson, D. (2020). Unifying access. Transportation Research Part D: Transport and Environment, 83(October 2019), 102355. https://doi.org/10.1016/j.trd.2020.102355

Xi, Y., Miller, E. J., \& Saxe, S. (2018). Exploring the Impact of Different Cut-off Times on Isochrone Measurements of Accessibility. Transportation Research Record, 2672(49), 113-124. https://doi.org/10.1177/0361198118783113

Yiannakoulias, N., Bland, W., \& Svenson, L. W. (2013). Estimating the effect of turn penalties and traffic congestion on measuring spatial accessibility to primary health care. Applied Geography, 39, 172-182. https://doi.org/10.1016/j.apgeog.2012.12.003

Zuluaga, J., \& Escobar, D. (2017). Análisis de accesibilidad territorial de la región noroccidente de Colombia. Revista Espacios, 38(6), 30-45.

Esta obra está bajo una Licencia Creative Commons Attribución-NoCommercial 4.0 International

\section{(c) $)$ BY-NC}

